\title{
The putative catalytic role of higher serotonin bioavailability in the clinical response to exposure and response prevention in obsessive-compulsive disorder
}

\author{
Thiago Sampaio, Cristiane Lima, Fabio Corregiari, Marcio Bernik \\ Programa Ansiedade (AMBAN), Instituto de Psiquiatria, Hospital das Clínicas da Faculdade de Medicina da Universidade de São Paulo \\ (FMUSP), São Paulo, SP, Brazil.
}

\begin{abstract}
Objective: Exposure and response prevention (ERP) is effective to treat obsessive-compulsive disorder (OCD), but the lack of tolerance to the aversion nature of exposure techniques results in a high drop-out rate. There have been reports of a generic stress endurance effect of serotonin (5-HT) in the central nervous system (CNS) which might be explained by suppression of defensive fixed action patterns. Previous studies have proposed that higher baseline 5-HT concentration and slow decrease in concentration during drug treatment of OCD were predictors of good clinical response to 5-HT reuptake inhibitors. The objective of this study was to investigate whether pre-treatment platelet rich plasma (PRP) 5-HT concentration is associated with latency of treatment response and final response to an ERP protocol for obsessive-compulsive disorder (OCD).

Methods: Thirty adult and treatment-free OCD patients were included in an 8-week, 16-session ERP protocol. 5-HT concentration was determined at baseline and after treatment. Patients with a reduction $\geqslant 30 \%$ on the Yale-Brown Obsessive Compulsive Scale (Y-BOCS) at the end of ERP were defined as responders.

Results: A positive correlation between baseline 5-HT concentration and reduction of symptoms on the Y-BOCS was observed after 4 weeks. Baseline 5-HT concentration was not correlated with clinical response after 8 weeks of ERP, possibly due to the similar though delayed clinical response of patients with lower (compared to those with higher) baseline 5-HT concentration. Patients with higher 5-HT baseline concentration also showed more improvement in depressive symptoms with treatment.

Conclusion: The present results partially support the hypothesis of a stress endurance effect of 5-HT in OCD patients. According to the literature, fast onset responders possibly have more or larger 5-HT containing neurons, higher endogenous 5-HT synthesis or lower monoamine oxidase activity; all these hypotheses remain to be investigated.
\end{abstract}

Keywords: Behavior therapy; biological markers; stress; neurophysiology; obsessive-compulsive disorder

\section{Introduction}

Behavior therapy focused on exposure and response prevention (ERP) is a first step treatment for obsessivecompulsive disorder (OCD) ${ }^{1,2}$ Nevertheless, treatment response is only around 60 to $83 \%,{ }^{3}$ and up to $25 \%$ of patients drop out prior to completion of treatment. ${ }^{4}$ This is likely due to lack of tolerance to the aversive nature of exposure techniques and to the slow rate of improvement. ${ }^{2}$

Considering that experiencing and mastering aversion during the exposure procedure is a core element of ERP effectiveness, the ability to identify better or faster responders prior to treatment would help the clinician choose between ERP or other non-aversive strategies. The best tool for identifying such patients would be ideally a biological variable that could be easily and reliably measured.

Correspondence: ThiagoSampaio, Rua Dr. Ovídio Pires de Campos, 785, Caixa Postal 3671, CEP 05403-903, São Paulo, SP, Brazil.

E-mail: thiagosa@usp.br

Submitted Apr 01 2015, accepted Dec 082015.
Serotonin (5-HT) has been reported to have a major impact on central nervous system (CNS) structures underlying stress endurance. ${ }^{5}$ The brain structures involved in both defense responses ${ }^{6}$ and obsessive-compulsive symptoms ${ }^{7}$ include some with the highest concentration of 5-HT containing neurons in the CNS. ${ }^{8}$ One possible mechanism for the observed stress endurance-enhancing properties of $5-\mathrm{HT}$ could be its suppressive effect of defensive fixed action patterns ${ }^{9}$ controlled by lower structures of the hierarchical defense system. ${ }^{10,11}$

The cognitive-behavioral approach, which emphasizes the influence of dysfunctional beliefs on emotional distress and compulsive behaviors, is currently the main psychological model of OCD. ${ }^{12}$ According to this model, compulsions are reinforced by short-term distress reduction and temporary removal of the intrusive thought, and prevent the individual from correct emotional processing ${ }^{13}$ and from learning that their appraisals are unrealistic. In turn, from an ethological perspective, compulsive rituals are exacerbations of primitive fixed action patterns. ${ }^{14}$ Thus, considering the biological, psychological, and ethological perspectives, it is possible to hypothesize that 
increased bioavailability of $5-\mathrm{HT}$ would boost corrective emotion processing ${ }^{13}$ by elevating the trigger threshold for fixed action patterns (i.e., compulsive responses), enabling different and more adaptive learned behaviors to emerge during ERP. Accordingly, patients with higher amounts of functionally active brain 5-HT would better endure exposure and achieve faster clinical response to ERP.

Pletscher \& Laubscher ${ }^{15}$ and Da Prada et al. ${ }^{16}$ have demonstrated the usefulness of using platelets as a model of CNS 5-HT functioning. In fact, over $99 \%$ of blood $5-\mathrm{HT}$ is contained in platelets, ${ }^{17}$ and $5-\mathrm{HT}$ concentration in platelet-rich plasma (PRP) is a valid marker of CNS 5 -HT bioavailability. ${ }^{18,19}$ Determination of $5-\mathrm{HT}$ in PRP is safer and less invasive than direct cerebrospinal fluid measurements, and cheaper than functional neuroimaging. ${ }^{20,21}$

Using whole blood 5-HT concentration, Humble et al. ${ }^{20}$ and Delorme et al. ${ }^{21}$ have proposed a higher baseline5HT concentration and a slow decrease in concentration during drug treatment of OCD as predictors of good clinical response to 5-HT reuptake inhibitors (SRIs). However, no studies so far have focused on the possible correlation between 5-HT function (including PRP 5-HT concentration) and clinical response to validated nonpharmacological treatments like ERP in OCD.

The purpose of this study was to investigate whether PRP 5-HT concentration is associated with the latency of clinical response and final clinical response to ERP treatment in OCD patients.

\section{Methods}

\section{Participants and procedures}

The study was carried out at Programa Ansiedade (AMBAN), Instituto de Psiquiatria, Hospital das Clínicas da Faculdade de Medicina da Universidade de São Paulo (FMUSP), São Paulo, SP, Brazil. Subjects were recruited from a sample of 88 individuals actively seeking treatment at our anxiety disorders program from February 2005 to July 2006. The inclusion criteria were: 1) meeting DSM-IV diagnostic criteria for OCD; and 2) age between 18 and 65 years. The exclusion criteria were: 1 ) history of psychotic symptoms or severe depression; 2) previous treatment with ERP techniques; 3) use of any drug that might interfere with 5-HT system or treatment response to ERP (i.e., sibutramine, selective serotonin reuptake inhibitor [SSRIs], SRIs, or benzodiazepines) in the 3 previous months. Before inclusion, subjects were screened by experienced psychiatrists during an intake session using the Structured Clinical Interview for DSM-IV-TR Axis I Disorders (SCID-I). ${ }^{22}$ Figure 1 shows the flow chart of patient enrollment.

Fifty-seven patients met all the inclusion criteria. These 57 patients were informed about the study design and the treatment procedures. The protocol consisted of 16 individual ERP sessions (90 minutes each), twice a week for 8 weeks (Table 1). No more than two absences were allowed during the 8-week period. On the third absence the patient was assumed to have dropped out from the study.
After the intake session, a further 27 patients were excluded: 16 began pharmacological treatments qualified as exclusion criteria (benzodiazepines in 5 patients and SRIs in 11 patients); two patients showed severe depressive symptoms (17-item Hamilton Rating Scale for Depression [HAM-D-17] $\geqslant 18)^{23}$; and nine patients refused to participate in the study stating the following reasons: non-acceptance of exposure procedures $(n=5)$, no time availability for two therapy sessions per week $(n=2)$, and no financial means to commute to the clinic twice a week $(n=1)$. Thus, 30 outpatients joined the ERP protocol. All ERP sessions and data collection occurred between August 2005 and December 2006.

\section{Efficacy measures}

A single independent rater (with no additional roles in the trial) blinded to $5-\mathrm{HT}$ measurements conducted all assessments of efficacy measures. Three assessments were performed: 1 week before the first ERP session (pretest); at the 4th treatment week (mid-treatment); and in the last session, at the 8th treatment week (end-treatment).

The primary efficacy measure selected for obsessivecompulsive symptoms was the total score of the YaleBrown Obsessive-Compulsive Scale (Y-BOCS). ${ }^{24}$ Patients who were found to have a reduction of $30 \%$ or greater in Y-BOCS score were defined as responders, whereas those with a reduction below $30 \%$ were defined as nonresponders The HAM-D-17 ${ }^{23}$ was used for assessment of depressive symptoms, and the Patient Rated Anxiety Scale (PRAS) ${ }^{25}$ for assessment of anxiety symptoms. Additionally, we measured the impact of treatment on patient functioning using the Sheehan Disability Scale. ${ }^{26}$ All measures have been empirically validated for Brazilian Portuguese and are widely used in clinical trials of psychopathological conditions.

\section{PRP 5-HT concentration analysis}

Blood was collected 1 week before treatment and upon the final therapy session. Blood was collected in $7.5-\mathrm{mL}$ EDTA tubes. Blood samples were centrifuged for $15 \mathrm{~min}-$ utes at $300 \times \mathrm{g}$. PRP was stored at $-80^{\circ} \mathrm{C}$ until dosage. Platelet count was performed using an automated cell counter (Beckman Coulter) for normalization of results.

5-HT concentration was measured with a Shimadzu $10 \mathrm{Vp}$ system in conjunction with a fluorescence detector (Shimadzu RF-10A XL). Standards for 5-HT were prepared by dissolving different amounts of $5-\mathrm{HT}$ hydrochloride (Sigma) in plasma.

\section{Statistical analysis}

Normality assumption was tested by the KolmogorovSmirnov test. Pearson's correlation test was used to verify correlations between PRP 5-HT (baseline and posttreatment concentrations) and treatment response based on the Y-BOCS percentage variation. Patients who were found to have a reduction of $30 \%$ or greater in Y-BOCS score were defined as responders, whereas those with a reduction below $30 \%$ were defined as non-responders. 


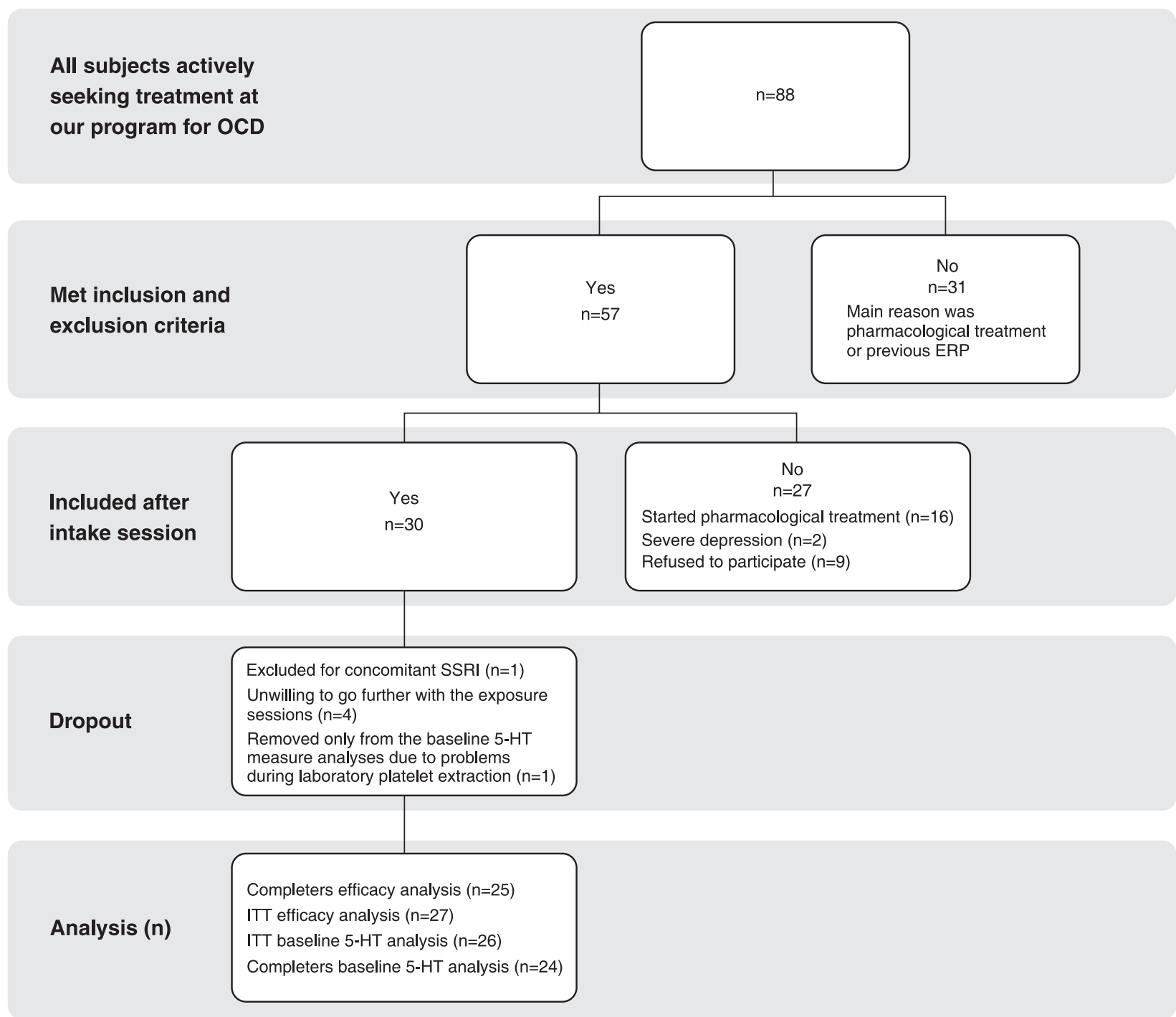

Figure 1 Flow chart of patient enrollment. 5-HT = serotonin; ERP = exposure and response prevention; ITT = intentionto-treat; $\mathrm{OCD}$ = obsessive-compulsive disorder; SSRI = selective serotonin reuptake inhibitor.

Treatment responders and non-responders were compared regarding other psychopathological measures using a t-test for independent samples. Significance was established as alpha $=5 \%$ in all cases .

\section{Ethics and protocol approval}

The study protocol is in accordance with the Declaration of Helsinki for research with human subjects and was approved by the Hospital Research Ethics Committee. All participants gave written informed consent.

\section{Results}

\section{Attrition}

Thirty patients started the treatment. One patient initiated concomitant SSRI treatment during the ERP protocol and was excluded from all analyses. Four additional patients
(13.8\%) were unwilling to proceed with the exposure sessions and dropped out. Two dropped out after mid-treatment and were included in the intention-to-treat (ITT) analysis $(n=27)$ using the last observation carried forward (LOCF). Completer analysis included 25 patients.

Data from one patient was removed from the baseline 5-HT sample analyses due to problems during laboratory platelet extraction. Thus, the sample size for the baseline 5 - $\mathrm{HT}$ concentration analysis was $\mathrm{n}=26$ on week 4 and $\mathrm{n}=24$ on week 8 .

\section{Sociodemographic and clinical characteristics of the sample}

Of the 28 patients who had their baseline $5-\mathrm{HT}$ concentration analyzed, 16 were female $(57.1 \%)$, 17 were single $(60.7 \%), 13$ had a college degree $(46.4 \%)$, and 14 were working at the time of the study $(50 \%)$. Mean age ( \pm SD) was $39.5 \pm 10.4$ years. 
Table 1 Summary outline of 16 sessions (8 weeks) of ERP for OCD

Session Session content

$1 \quad$ A) Therapeutic contract (rules and commitments).

B) Psychoeducational explanation concerning relevant OCD information.

C) Rationale of behavioral treatment and ERP procedures: gradual hierarchical exposure, habituation, usage of Subjective Units of Distress Scale (SUDS).

D) Completing the "List of obsessions and (mental and motor) rituals."

E) Determination of the target area (focus) for ERP.

F) Homework: reading the psychoeducational material and writing "OCD-symptom weekly diary."

2 A) Check for observations or pending issues regarding the previous session.

B) Homework check and review of exposure and habituation concepts.

C) Elaboration of exposure hierarchy.

D) Therapist-assisted exposure to first item of hierarchy.

E) Completing the "self-exposure diary" with the patient and providing guidance on how to record all out-of-session exposure exercises on the next hierarchy item.

F) Homework: self-controlled exposure with self-imposed response prevention to the same item applied in the session.

3 to 15 A) Check for observations or pending issues regarding previous session.

B) Check for possible symptoms to include in the "List of obsessions and (mental and motor) rituals."

C) Checking homework and evaluating if the self-exposure diary was correctly completed.

D) Review of exposure hierarchy and elaboration of new items and/or focus if necessary.

E) Selection of two new hierarchy items.

F) Application of ERP to one or more of the selected items.

G) Completing the "self-exposure diary" with the patient and providing guidance on how to record all out-of-session exposure exercises on the next hierarchy item.

H) Homework: self-controlled exposure with self-imposed response prevention (ERP).

16 A) Final evaluation: preparation for monthly follow-up.

B) Discussion about ways to cope with setbacks and strategies for the maintenance of goals.

$\mathrm{ERP}=$ exposure with response prevention; $\mathrm{OCD}=$ obsessive-compulsive disorder.

Considering all the 30 patients who initiated the trial, the mean age at onset of obsessive symptoms was $15.9 \pm 8.6$ years, $16.4 \pm 8.7$ years for compulsive symptoms, 24.6 \pm 10.2 years for perception of impairment, and $34.5 \pm 12.2$ years for treatment seeking. The most common psychiatric comorbidity was depression $(n=3)$, followed by social phobia $(n=2)$, simple phobia $(n=2$, one patient with animal subtype, one with claustrophobia) and bipolar spectrum disorder $(n=1)$.

Only three patients had undergone previous SRI or SSRI treatment.

\section{Efficacy analysis}

Considering the 25 completers, 17 (68\%) patients responded to treatment (reduction of $30 \%$ or greater in Y-BOCS score), whereas 8 (32\%) did not respond. Responders showed higher decreases in generalized anxiety (PRAS) $(t=-2.88 \mathrm{p}=0.01)$ and family disability $(t=-3.75 \mathrm{p}=$ 0.01 ) severity scores (Sheehan Scale), whereas no differences were found between the groups regarding the change scores recorded for work and social disability (Sheehan Scale) and depressive symptoms severity (HAM-D-17).

The two drop-out patients who were included in the ITT analysis did not respond to treatment. Therefore, $10(37 \%)$ patients were considered as non-responders, whereas the number of responders remained the same (17 patients, 63\%) as that of the completer analysis (95\% confidence interval $[95 \% \mathrm{Cl}]$ 0.45-0.81). The finding of more pronounced decreases in generalized anxiety $(t=-2.64 \mathrm{p}=0.01)$ and family disability $(t=-3.05 \mathrm{p}=0.01)$ severity scores detected by completer analysis was maintained in ITT analysis.

\section{PRP 5-HT concentration and clinical outcome}

Baseline 5-HT concentration was not correlated with clinical improvement (i.e., percentage Y-BOCS change from baseline) after 8 weeks of ERP $(r=-0.29 ; p=0.14)$. Nevertheless, higher baseline 5-HT concentration correlated with more pronounced decrease in Y-BOCS scores (percentage change from baseline) after 4 weeks of ERP $(r=-0.42 ; p=0.03)$ (Figure 2).

A correlation between higher baseline PRP 5-HT concentration and decrease in depression symptom severity (HAM-D-17) was found after 8 weeks, both in completer $(r=-0.42 ; p=0.04)$ and ITT samples analyses $(r=-0.45$; $\mathrm{p}=0.02$ ) (Figure 3).

Table 2 summarizes the descriptive statistics for baseline 5-HT and Y-BOCS variations.

\section{Discussion}

The relatively broad range of clinical response rates reported in the literature (60 to $83 \%$ ) for ERP might be explained by differences in the response criteria adopted by each study. Although most studies have used percent reduction in the Y-BOCS as the clinical response criteria, the cutoff point for response has varied from 20 to $50 \%$ reduction in Y-BOCS score. A review ${ }^{27}$ focusing on this issue has proposed that a reduction $\geqslant 30 \%$ in $\mathrm{Y}$-BOCS score after treatment appears to be optimal for determining clinical improvement. This cutoff point has thus been 


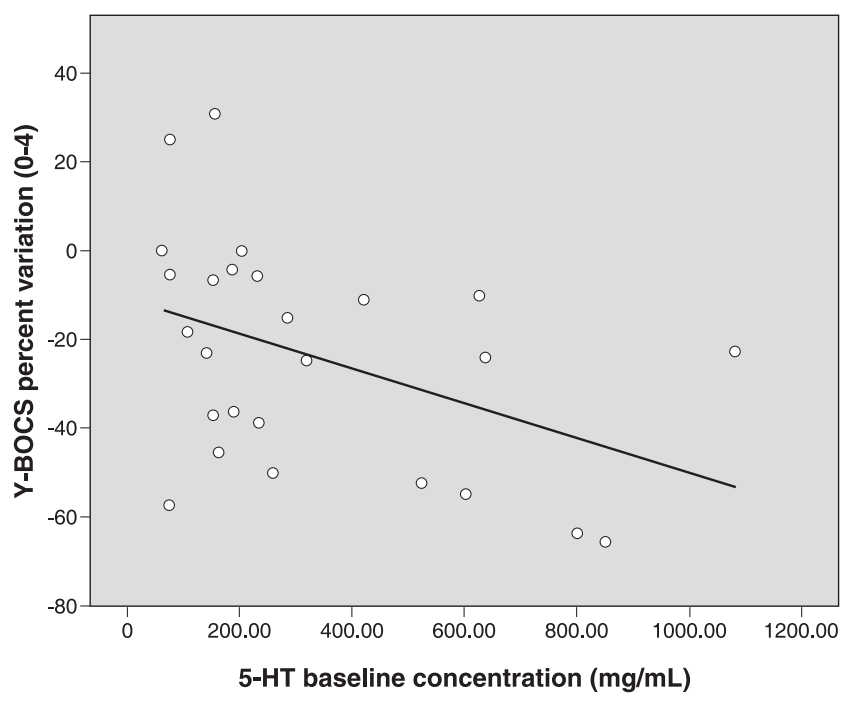

Figure 2 Correlation between serotonin (5-HT) baseline concentration $(\mathrm{mg} / \mathrm{mL})$ and Yale-Brown Obsessive-Compulsive Scale (Y-BOCS) percentage variation after 4 weeks of exposure and response prevention therapy (mid-test).

employed as a standard definition of treatment response in order to facilitate cross-study comparisons. Thus, despite of a relatively large confidence interval for percent of responders $(95 \% \mathrm{Cl} 0.45-0.81)$, the ERP efficacy in this trial can be considered average compared with the rate of clinical response reported by Lewin et al. ${ }^{3}$

Similar findings were obtained with completer and ITT analysis. This suggests the absence of attrition bias. Responders had higher decreases in generalized anxiety and family disability severity scores (completer and ITT analysis). These results were expected, considering that anxiety habituation is the focus of exposure exercises and OCD rituals are often related to family disability. Possibly, the lack of difference between groups regarding work and social disability is due to the fairly low rate of participants working during treatment period (50\%).

To the best of our knowledge, this is the first study investigating PRP 5-HT concentration as a predictor of outcome in patients treated with psychotherapy. In the studied sample, OCD patients with higher baseline PRP 5-HT concentration experienced faster onset of clinical response (at 4 weeks) to ERP. Interestingly, baseline 5-HT concentration was not correlated with clinical response at week 8 , in spite of sustained clinical improvement of all patients that responded to ERP at week 4. The main hypotheses are: 1) the better clinical response in higher baseline $5-\mathrm{HT}$ concentration patients would be sustained at week 8 in a larger sample; or 2) despite increased stress endurance of those patients with higher baseline $5-\mathrm{HT}$, this advantage might have been overcome by other non-biochemical factors (e.g., good therapeutic alliance). Other larger sample and well-designed studies are needed to confirm these hypotheses. In addition, we observed that higher baseline 5-HT concentrations also predicted decrease of depressive symptoms after 8 weeks of ERP.

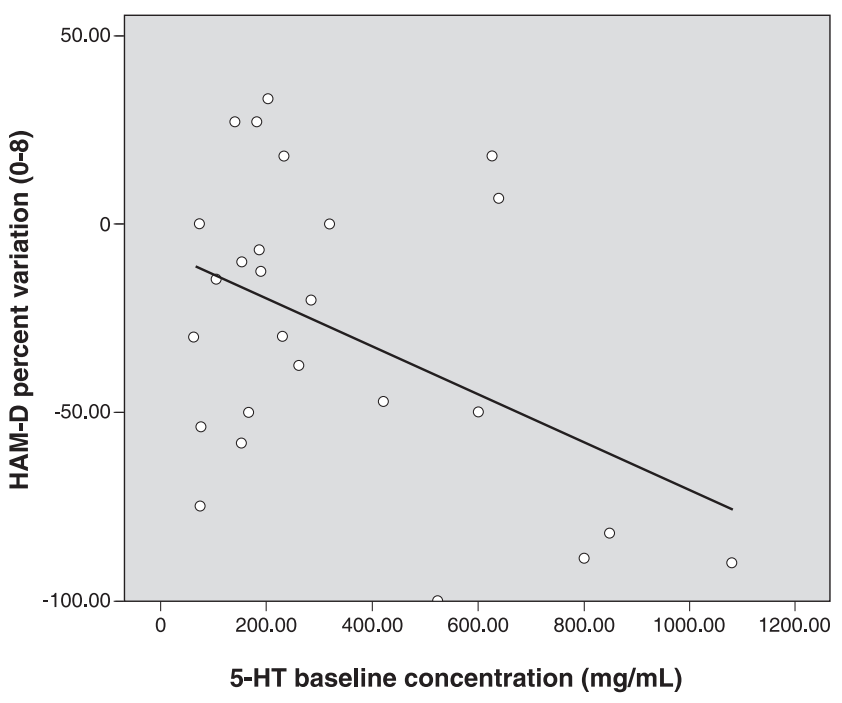

Figure 3 Correlation between serotonin (5-HT) baseline concentration $(\mathrm{mg} / \mathrm{mL})$ and percent change in 17-item Hamilton Rating Scale for Depression (HAM-D-17) score after 8 weeks of exposure and response prevention therapy (intention-to-treat sample analysis).

The higher baseline concentration of $5-\mathrm{HT}$ in patients with faster onset of clinical response found in this sample is consonant with the results reported by both Humble et al. ${ }^{20}$ and Delorme et al. ${ }^{21}$ regarding whole blood 5-HT concentration measurements and SRIs treatment. These similarities suggest a role of $5-\mathrm{HT}$ in regulating the timing of improvement following both pharmacological and behavioral treatments. Conversely, despite previous reports of similar post-treatment effects of both SRIs and ERP on normalization of glucose metabolism in successfully treated OCD patients, ${ }^{28}$ we did not observe a decrease in 5 -HT concentration as reported by Humble et al. ${ }^{20}$ and Delorme et al. ${ }^{21}$

Humble et al. ${ }^{20}$ and Delorme et al. ${ }^{21}$ hypothesized that a more active 5-HT transporter in faster onset responders would explain the higher 5-HT platelet concentration and faster decreases following SRI treatment. However, in the present study, in which there was no pharmacological manipulation of the 5-HT system, we hypothesize that patients with faster onset of response had a higher baseline pool of bioavailable 5-HT resulting from the presence of more or larger 5-HT containing neurons, higher endogenous 5-HT synthesis or lower monoamine oxidase activity, all of which remain to be investigated.

The present study results are in accordance with Graeff's ${ }^{5,29}$ postulated roles of $5-\mathrm{HT}$ in modulating the brain defense systems. 5-HT would enhance learned responses to distal threat expressed by the forebrain while inhibiting unconditioned responses (e.g., primitive fixed action patterns) to proximal threat expressed by the periaqueductal gray matter (PAG).

According to this model, higher 5-HT bioavailability would enhance emotional processing (i.e., habituation) ${ }^{13}$ during exposure, while undermining defensive fixed action patterns such as rituals in threatening situations. ${ }^{5}$ Conversely, an intrinsically reduced $5-\mathrm{HT}$ bioavailability 
Table 2 Descriptive statistics (intention-to-treat sample).

\begin{tabular}{lccc}
\hline & $\begin{array}{c}\text { Baseline 5-HT } \\
(\mathrm{mg} / \mathrm{mL})\end{array}$ & $\begin{array}{c}\text { Y-BOCS \% } \\
\text { variation }(0-4)\end{array}$ & $\begin{array}{c}\text { Y-BOCS \% } \\
\text { variation }(0-8)\end{array}$ \\
\hline $\mathrm{n}$ & $26^{*}$ & 27 & 27 \\
Mean & 326.08 & -20.70 & -40.44 \\
Standard error of mean & 54.50 & 5.08 & 6.38 \\
Standard deviation & 277.90 & 26.43 & 33.16 \\
Range & $1,018.00$ & 96.29 & 122.73 \\
Minimum & 62.75 & -66 & -100 \\
Maximum & $1,080.75$ & 31 & $22.73^{\dagger}$ \\
Percentile 25 & 150.46 & -45.45 & -61.11 \\
Median & 196.05 & -15.00 & -36.36 \\
Percentile 75 & 543.05 & 0.00 & -15 \\
\hline
\end{tabular}

5-HT = serotonin; Y-BOCS = Yale-Brown Obsessive-Compulsive Scale.

* One patient was removed from the baseline 5-HT sample analyses due to problems during laboratory platelet extraction. Therefore, baseline 5-HT analysis included 26 patients.

Only two patients had higher Y-BOCS scores after treatment, with 8.33 and $22.73 \%$ increases in Y-BOCS total scores.

might impair the tolerance of some individuals to aversive states such as those induced by exposure exercises, restraining the learning of alternative skills to cope with aversive situations without eliciting primitive fixed action patterns, such as stereotyped compulsive rituals. ${ }^{30}$

In view of this reasoning, the prediction that patients with more bioavailability of $5-\mathrm{HT}$ would endure ERP treatment better and show faster and stronger clinical improvements was partially supported by the present study results. Alternatively, and as a study limitation, partially diverging results between the present study and previous ones using SRIs might be explained by the lack of a control group (without treatment) and the relatively small sample size. Nevertheless, it is presently difficult to enroll larger samples of adults who are not users of SRIs. This is true even in large urban OCD treatment centers; it is increasingly more difficult to find non-pediatric OCD patients not currently using SRIs and without a history of exposure-based treatments.

Although our findings suggest that higher baseline 5-HT concentration predicts faster clinical onset of response to ERP for OCD symptoms and improvement of depressive symptoms, it is too soon to start dosing PRP 5-HT concentration before treating OCD patients with ERP. Given the pioneering scope and the small sample size of this study, more studies, ideally with larger samples and using untreated control groups, are needed. Nevertheless, if these findings are confirmed, PRP 5-HT concentration may be a promising, cheap, reliable and feasible predictor of outcome to exposure-based treatments in OCD.

\section{Acknowledgements}

We thank Prof. Wagner Gattaz, Leda Talib, and the LIM23 staff for the 5-HT/PRP analyses; Maria Cláudia Bravo for designing the ERP protocol; and Daniele Tartuce and Jane Cleide Andrade da Rosa for their assistance with the treatment of some patients. We are also thankful to Clóvis Sousa, Eduardo Nakano, Helton Graziadei, and Eriton Barros for the statistical analysis; to Luciana dos Santos and Gustavo Pimenta for providing secretarial support; and to all AMBAN colleagues who have contributed with comments on the manuscript.

\section{Disclosure}

The authors report no conflicts of interest.

\section{References}

1 Ponniah K, Magiati I, Hollon SD. An update on the efficacy of psychological therapies in the treatment of obsessive-compulsive disorder in adults. J Obsessive Compuls Relat Disord. 2013;2: 207-18.

2 National Collaborating Centre for Mental Health. Obsessivecompulsive disorder: core interventions in the treatment of obsessivecompulsive disorder and body dysmorphic disorder. Leicester: British Psychological Society; 2006.

3 Lewin AB, Wu MS, McGuire JF, Storch EA. Cognitive behavior therapy for obsessive-compulsive and related disorders. Psychiatr Clin North Am. 2014;37:415-45.

4 Lack CW. Obsessive-compulsive disorder: evidence-based treatments and future directions for research. World J Psychiatry. 2012;2:86-90.

5 Graeff FG. On serotonin and experimental anxiety. Psychopharmacology (Berl). 2002;163:467-76.

6 Mobbs D, Petrovic P, Marchant JL, Hassabis D, Weiskopf $N$, Seymour B, et al. When fear is near: threat imminence elicits prefrontal-periaqueductal gray shifts in humans. Science. 2007;317: 1079-83.

7 Dold M, Aigner M. [Neuroimaging the various symptom dimensions of obsessive-compulsive disorder]. Neuropsychiatr. 2009;23: 193-205.

8 Murphy DL, Moya PR, Fox MA, Rubenstein LM, Wendland JR, Timpano KR. Anxiety and affective disorder comorbidity related to serotonin and other neurotransmitter systems: obsessive-compulsive disorder as an example of overlapping clinical and genetic heterogeneity. Philos Trans R Soc Lond B Biol Sci. 2013;368:20120435.

9 McNaughton N, Andrews G. Anxiety. Dunedin: University of Otagosemi; 1990.

10 Graeff FG, Guimarães FS, De Andrade TG, Deakin JF. Role of 5-HT in stress, anxiety, and depression. Pharmacol Biochem Behav. 1996;54:129-41.

11 Gray JA, McNaughton N. The neuropsychology of anxiety: an enquiry into the functions of the septo-hippocampal system. 2nd ed. Oxford: Oxford University; 2000.

12 Abramowitz JS, Taylor S, McKay D. Obsessive-compulsive disorder. Lancet. 2009;374:491-9.

13 Foa EB, Kozak MJ. Emotional processing of fear: exposure to corrective information. Psychol Bull. 1986;99:20-35.

14 Rapoport JL. Recent advances in obsessive-compulsive disorder. Neuropsychopharmacology. 1991;5:1-10.

15 Pletscher A, Laubscher A. Blood platelets as models for neurons: uses and limitations. J Neural Transm Suppl. 1980;16:7-16.

16 Da Prada M, Cesura AM, Launay JM, Richards JG. Platelets as a model for neurones? Experientia. 1988;44:115-26. 
17 Celada P, Martín F, Artigas F. Effects of chronic treatment with dexfenfluramine on serotonin in rat blood, brain and lung tissue. Life Sci. 1994;55:1237-43.

18 Bianchi M, Moser C, Lazzarini C, Vecchiato E, Crespi F. Forced swimming test and fluoxetine treatment: in vivo evidence that peripheral 5-HT in rat platelet-rich plasma mirrors cerebral extracellular 5-HT levels, whilst 5-HT in isolated platelets mirrors neuronal 5-HT changes. Exp Brain Res. 2002;143:191-7.

19 Koudouovoh-Tripp P, Sperner-Unterweger B. Influence of mental stress on platelet bioactivity. World J Psychiatry. 2012;2:134-47.

20 Humble M, Bejerot S, Bergqvist PB, Bengtsson F. Reactivity of serotonin in whole blood: relationship with drug response in obsessivecompulsive disorder. Biol Psychiatry. 2001;49:360-8.

21 Delorme R, Chabane N, Callebert J, Falissard B, Mouren-Siméoni $\mathrm{MC}$, Rouillon F, et al. Platelet serotonergic predictors of clinical improvement in obsessive compulsive disorder. J Clin Psychopharmacol. 2004; 24:18-23.

22 First MB. Structured clinical interview for DSM-IV-TR Axis I disorders: patient editionNew York: Columbia University; 2005.

23 Hamilton M. A rating scale for depression. J Neurol Neurosurg Psychiatry. 1960;23:56-62.
24 Goodman WK, Price LH, Rasmussen SA, Mazure C, Delgado P, Heninger GR, et al. The Yale-Brown Obsessive Compulsive Scale. II. Validity. Arch Gen Psychiatry. 1989;46:1012-6.

25 Sheehan DV, Lecrubier Y, Sheehan KH, Amorim P, Janavs J, Weiller E, et al. The Mini-International Neuropsychiatric Interview (M.I.N.I.): the development and validation of a structured diagnostic psychiatric interview for DSM-IV and ICD-10. J Clin Psychiatry. 1998;59:22-33;quiz 4-57.

26 Sheehan DV, Harnett-Sheehan K, Raj BA. The measurement of disability. Int Clin Psychopharmacol. 1996;11:89-95.

27 Tolin DF, Abramowitz JS, Diefenbach GJ. Defining response in clinical trials for obsessive-compulsive disorder: a signal detection analysis of the Yale-Brown obsessive compulsive scale. J Clin Psychiatry. 2005;66:1549-57.

28 Baer L. Behavior therapy: endogenous serotonin therapy? J Clin Psychiatry. 1996;57:33-5.

29 Graeff FG. Neuroanatomy and neurotransmitter regulation of defensive behaviors and related emotions in mammals. Braz $\mathrm{J}$ Med Biol Res. 1994;27:811-29.

30 Hewlett WA, Vinogradov S, Martin K, Berman S, Csernansky JG. Fenfluramine stimulation of prolactin in obsessive-compulsive disorder. Psychiatry Res. 1992;42:81-92. 